\title{
Multi-Layer Magnetic Focusing Sensor Structure for Pulsed Remote Field Eddy Current
}

\author{
Changrong Yang ${ }^{1}$, Bin $\mathrm{Gao}^{1 *}$, Qiuping $\mathrm{Ma}^{1}$, Lian Xie ${ }^{1}$, Guiyun Tian ${ }^{1,2}$, Ying Yin $^{3}$ \\ ${ }^{1}$ School of Automation Engineering, University of Electronic Science and Technology of China, China \\ ${ }^{2}$ School of Engineering, Newcastle University, England, UK \\ ${ }^{3}$ Sichuan Special Equipment Inspection Institute, China \\ * Corresponding author: bin_gao@uestc.edu.cn
}

\begin{abstract}
Pulsed Remote field eddy current (PRFEC) technique has been utilized broadly in detection of tubular material because of its abundant frequency spectrums with lower power consumption. A novel multi-layer magnetic focusing sensor structure is proposed for PRFEC. The new sensing structure consists of several promising characteristics. It significantly enhances the detectability of the deeper depth of ferromagnetic material, and provides detection capability without covering $U$ type component. Both driver and receiver coil are integrated in the same sensing structure while the traditional methods are put them separated. The theoretical derivation based on electromagnetic principles has been developed for analyzing and interpreting the results. In addition, number of experiments and simulations on different defects have been studied. In experiments, both surface and subsurface defects with different width, depth, inclined angle and circular type have been carried out. The results have confirmed that all types of defects can be detected and it has shown the relatively preferable linear relationships and reliabilities.
\end{abstract}

Index Terms- Remote field eddy current, magnetic shielding, ferromagnetic plate, pulse excitation.

\section{INTRODUCTION}

$\mathrm{E}$ LECTROMAGNETIC nondestructive testing (ENDT) methods have been broadly used for inspection of conductive material in industry [1]. Alternating current field measurement (ACFM) technique is a promising method which can straightly obtain the information of defects by measuring the two magnetic field components. Lift-off effect in ACFM can be avoided and it is mainly applied to detect the gas pipelines and rails [2-5]. Unfortunately, ACFM is difficult to detect a deeper flaw because of skin-effect, and uniform induced current is required in ACFM. This will reduce the area of detection due to local uniform induced current [6-7]. Magnetic flux leakage (MFL) is usually comprised of direct-current excitation or permanent magnet and ferrite core as it is sensitive for ferromagnetic defects. MFL related research works have been conducted in defects characteristic classifications. In particular, these features include arrival time of the inflexion point, signal magnitude and phase variation of the frequency components, pulse excitation and magnetic resistive sensor [8-12]. However, MFL cannot inspect a non-magnetic material and require magnetization of the specimen under the test [13]. Conventional eddy current testing (ECT) is limited to only inspect the defects of surface and near-surface region, it is difficult to detect deeper defects due to the skin-effect [1]. Remote field eddy current testing (RFECT) breaks through this limitation of detection depth, which typical features of "potential valley" and "phase knot", have been mainly applied for inspection of metal pipelines. There is identical sensitivity on both inside and outside of pipes' defects and the receiver coil is away from the driver coil with 2 3 inner diameters [14-16].
W. Lord et al. simulated RFECT phenomenon in ferromagnetic pipe using finite element method (FEM) [16]. H. Hoshikawa et al. derived the intensity of the electromagnetic energy flow as well as its direction to elucidate the remote field phenomena [17]. H. Fukutomi $e t$ $a l$. used RFECT to test steam generator tubes [15]. D. Kim et al. utilized RFECT for detection of the corrosion cracks in gas transmission pipelines [18]. M. Chen et al. described the response of pulsed remote eddy current testing (PRFECT) in the tube by using FEM as the response is not interval between the rising edge and trailing edge [19]. BF. Yang et al. proposed PRFECT array technique contained 4 sensing coils with $90^{\circ}$ interval for detection of ferromagnetic pipes, pulse excitation has an abundant frequency spectrums which can carry more information of the tested pieces. In addition, the characteristics of RFECT in pulse excitation was studied, the magnetic field line was restrained in a vicinity of the driver coil in the duration time A zero-crossing time was considered as feature for RFEC a linear relationship with depths of cracks [20-21]. XJ. Xu et $a l$. presented a novel orthogonal magnetic field excitation, a cylindrical conductor was inserted into a spiral coil and driven with current individually. This proposed probe could inspect radial and axial defects [22]. QW. Luo extracted zero-crossing time of PRFECT to analyze the thickness of different pipe with Least Squares Support Vector Regression [23]. D. Vasic applied PRFECT to measure the wall thickness of ferromagnetic tube. In addition, D Vasic found that a low frequency excitation is unable to discern whether the defect exists in external or internal. However, the internal or external cracks could be identified with shorter distance between the coils and high drive frequency [24-26]. M. Dadic et al. studied both time and frequency domain features using system identification to acquire a better model of PRFECT [27]. The Fourier filtering, crosscorrelation and wavelet transform techniques were applied to process RFECT signal under the inspection of the bend regions of steam generator. It illustrated the Fourier filtering had the effective noise reduction capability while the cross-correlation enhanced significantly the amplitude of the defect signal. In addition, the wavelet transform technique revealed the capability of signal enhancement as well as noise reduction [28].

There are less reports of RFECT for detecting conductive plates. Two primary methods are used to produce RFEC phenomenon, one is to employ u-type component (UTC), and another is to use magnetic shielding directly between the excitation and sensing coil. N. Kasai et al. proposed UTC to simulate RFEC in pipe for evaluating back-side flaws of the bottom plates of an oil-storage tank, and the structure of pick-up coil with u type ferrite core was 
investigated to reduce the distance between the driver and the receiver coil [29-30]. J. Wang made several comparisons between RFECT applied UTC and low frequency eddy current testing (LFECT) [31]. BF. Yang employed PRFECT with UTC to study non-magnetic flat conductive plates, it was efficient for shortening the distance of the driver coil and the pick-up coil by exerting magnetic shielding and ferrite core [32]. N. Kobayashi et al. reported the flux guide to increase the radial magnetic field intensity and decrease the direct magnetic field around the central axis of the tube to improve the sensitivity [33]. Application of magnetic shielding techniques [34-37] can enhance the strength of the receiver coil and suppress the perturbation of the primary magnetic field. Young-Kil Shin et al. proposed a shielding shape encircling RFEC probe for inspection of the nuclear fuel rods. The RFEC phenomenon produced by rods covered with probe and shielding parts. The results illustrated a linear relationship between phase of pick-up signals and depth of defects [38]. DQ. Zhou studied mainly influence of the metallic shields against PEC probe for ferrite-magnetic materials [39]. ZH. Liu et al. applied double excitation on magnetic shielding to detect sub-surface defects for stainless steel [40]. Y. Sun applied the RFECT to inspect metallic conductive plates. Pot core and auxiliary coil are involved and aluminum is covered to decrease the magnetic flux leakage under the plate [41]. A thick-walled aluminum up to $25.4 \mathrm{~mm}$ could be detected in the investigation from Y. Sun and L. Upda, yet the dimensions of probe was rather bigger. The outer diameter of the excitation coil was up to $10 \mathrm{~cm}$ and distance from driver coil to receiver coil was approximately $13 \mathrm{~cm}$ [42]. HT. Wang et al. designed a RFCET probe with three coils embedded in parts of shields, the energy penetrated the plate twice [43].

However, structure of RFEC using UTC whose size is bigger and that reduce the resolution, and the material of UTC will be changed with the change of testing material. In addition, the paper [41-43] studied energy flow with different shielding materials, as the auxiliary coils increase the complexity of the system. Furthermore, the distance from the driver coil to the pick-up coil is far. In this paper, we design a multi-layer magnetic focusing sensor structure for RFEC based on energy shielding theory. Conventional RFEC has been mainly aimed for the detection of tubular components, multi-layer magnetic shielding structured RFEC testing for inspection of flat plate has been studied in this paper. In the proposed RFEC profile, both driver and pick-up coil are coaxial where this is different from the conventional RFEC where driver and pick-up coil should be segregated with a certain distance. The shielding structures adopted are distinguished from previous UTC structure as the advantages can be drawn the shielding material are not required to be the same as the testing material. Thus it provides potential ability to be expanded in wider applications. Notwithstanding above, compared with general eddy current methods, the proposed method has deeper penetration depth especially for ferromagnetic material. Excitation in a pulse which can reduce energy loss, and obtain rich frequency spectrum for sub-surface defects detection.

The paper is organized as follows: Section II discusses the theoretical model of the proposed RFEC system. The results and discussion are presented in Section III. Finally, conclusions and further work are outlined in Section IV.

\section{METHODOLOGY}

\section{A. Principle of RFEC in the plate and magnetic shielding mechanism}

The conduction of RFEC in the plate is based on the principle of electromagnetic energy diffusion, the energy travels through the planar conductive plate from the surface into the bottom of the plate and return back to the surface. In the return process, the information of the flat conductor who contains the defects will be carried.

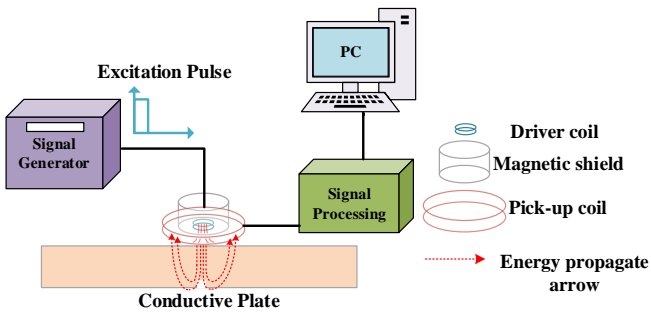

Fig. 1 Diagram of RFEC system

Fig. 1 shows the block diagram of the RFECT. Because the energy (Poynting Vector) produced by coil diffuses around the driver (blue and red arrow line in Fig. 1), the indirect coupling energy who carries the flaw message is very weak when return back to the surface of plate. If the direct coupling energy in the plate is more than the indirect energy, it will hinder the indirect coupling energy. Therefore, in order to inspect the defects in the conductive plate, we adopt the magnetic shielding units to suppress the direct coupling energy.

The magnetic shielding is applied to obtain maximum attenuation with the shield configuration who made of a high conductive or a high permeability materials. The result of shielding depends on the shield material, source field frequency and geometry. With the eddy current effect of a highly conductive shield, there will occur an opposing induced magnetic field by the eddy current on the surface when the source magnetic field flows into the shield. The induced magnetic field can mitigate the source field for achieving the magnetic shielding. With a high permeability material to shield, the magnetic resistance is smaller than that in the air. The magnetic field flows along the direction of a relatively lower magnetic resistance and the result of shielding is usually described by shielding effectiveness $(S E)$, namely

$$
S E=20 \log \frac{\left|B_{0}\right|}{\left|B_{S}\right|}[d B]
$$

where $B_{0}$ is the magnetic flux density at the measurement point without the shield, and $B_{s}$ is the magnetic flux density with the shield.

\section{B. The proposed sensor structure}

Multilayer magnetic focusing structure is designed to shield the energy above the conductive plate. According to the Maxwell equations and the constitutive relation, the probe model is symmetrical so that there is only a circumferential component who can decrease the computational time. Fig. 2 illustrates the proposed model, the impaction of different number of shielding layers have been investigated. One or two layer do not generate RFEC phenomenon even though the sizes, shielding materials and spacing have been altered. Three and four or more layers conduct the characteristic of RFEC whereas the three layers 
has been proved to give an excellent signal strength and optimal dimensions. Therefore, three layers shielding model is used for theoretical analysis. The study suppose that the medium is isotropic, linear, and homogeneous. Due to: $\nabla \cdot B=0$, the magnetic vector potential $A$ is introduced to solve conveniently.

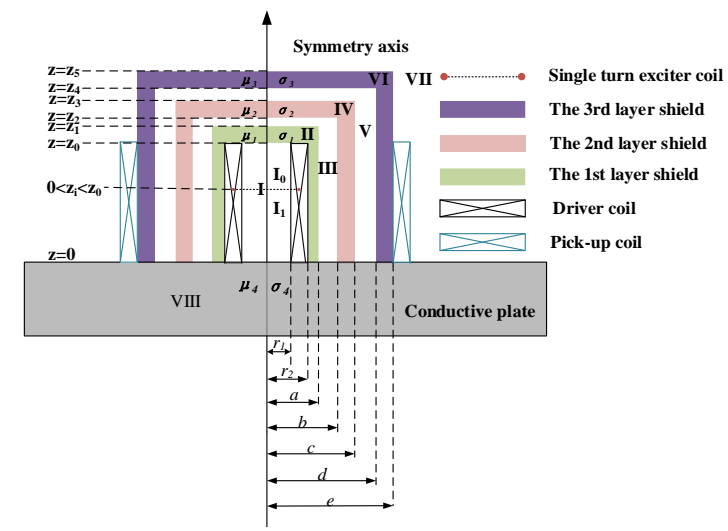

Fig. 2 RFEC probe proposed

$$
\begin{gathered}
B=\nabla \times A \\
\because \nabla \times H=J+J_{e}+\frac{\partial D}{\partial t}
\end{gathered}
$$

where $J$ is induced eddy current density, $J_{s}$ is source current density of driver coil and $D$ is displacement current density who can be ignored when the driver frequency is low frequency, the term $\frac{\partial D}{\partial t}$ will be omitted, namely

$$
\begin{gathered}
\frac{1}{\mu} \nabla \times B=J+J_{e} \\
\frac{1}{\mu} \nabla \times(\nabla \times A)=\frac{1}{\mu}\left(\nabla(\nabla \cdot A)-\nabla^{2} A\right)=J+J_{e}
\end{gathered}
$$

According to Coulomb gauge $\nabla \cdot A=0$

$$
-\nabla^{2} A=J+J_{e}
$$

In cylindrical coordinate, there is only a circumferential component, $A$ is a function of $r$ and $z$.

Therefore, Equation (6) is simplified as

$$
\begin{aligned}
& \nabla^{2} A=e_{\theta}\left(\nabla^{2} A_{\theta}-\frac{A_{\theta}}{r^{2}}+\frac{2}{r^{2}} \frac{\partial A_{r}}{\partial \theta}\right) \\
& =e_{\theta}\left(\frac{1}{r} \frac{\partial}{\partial r}\left(r \frac{\partial A_{\theta}}{\partial r}\right)+\frac{1}{r^{2}} \frac{\partial^{2} A_{\theta}}{\partial \theta^{2}}+\frac{\partial^{2} A_{\theta}}{\partial z^{2}}-\frac{A_{\theta}}{r^{2}}+\frac{2}{r^{2}} \frac{\partial A_{r}}{\partial \theta}\right) \\
& \text { Hence } \frac{\partial^{2} A_{\theta}}{\partial \theta^{2}}=0, \frac{\partial A_{r}}{\partial \theta}=0 . \\
& \therefore \nabla^{2} A=e_{\theta}\left(\frac{1}{r}\left(\frac{\partial A_{\theta}}{\partial r}+r \frac{\partial^{2} A_{\theta}}{\partial r^{2}}\right)-\frac{A_{\theta}}{r^{2}}+\frac{\partial^{2} A_{\theta}}{\partial z^{2}}\right)
\end{aligned}
$$

In order to express the conveniently, $A_{\theta}$ is replaced as $A$ in ultimate formulation, namely

$$
\begin{gathered}
\therefore \frac{1}{\mu} \nabla^{2} A=\frac{1}{\mu}\left(\frac{\partial^{2} A}{\partial r^{2}}+\frac{1}{r} \frac{\partial A}{\partial r}+\frac{\partial^{2} A}{\partial z^{2}}-\frac{A}{r^{2}}\right)=-J+\sigma \frac{\partial A}{\partial t} \\
\therefore \frac{\partial^{2} A}{\partial r^{2}}+\frac{1}{r} \frac{\partial A}{\partial r}+\frac{\partial^{2} A}{\partial z^{2}}-\frac{A}{r^{2}}=-J+\sigma \frac{\partial A}{\partial t}
\end{gathered}
$$

Above detail formula derivation can be regarded as governing equation of axisymmetric RFEC, the current density $J$ could be ignored in governing equation and regarded as boundary condition between driver coil and shielding layers. The propagation of electromagnetic field is related the conductivity and permeability of the material which influences both direction and property of propagation. Therefore, the boundary from different shielding layers are utilized to depict better constraint conditions when electromagnetic field propagates into the different mediums.

Region I/III/V/VII:

$$
\frac{\partial^{2} A}{\partial r^{2}}+\frac{1}{r} \frac{\partial A}{\partial r}+\frac{\partial^{2} A}{\partial z^{2}}-\frac{A}{r^{2}}=0
$$

According to the method of separation of variables [46]:

$$
A=\int_{0}^{\infty}\left[B e^{\alpha z}+C e^{-\alpha z}\right]\left[D J_{1}(\alpha r)+E Y_{1}(\alpha r)\right] d \alpha
$$

\section{Region II/IV/VI/VIII:}

$$
\frac{\partial^{2} A}{\partial r^{2}}+\frac{1}{r} \frac{\partial A}{\partial r}+\frac{\partial^{2} A}{\partial z^{2}}-\frac{A}{r^{2}}-j w \mu_{i} \sigma_{i} A=0(i=1,2,3,4)
$$

In the same way,

$$
\begin{aligned}
A=\int_{0}^{\infty}\left[B e^{\alpha z}+C e^{-\alpha z}\right]\left[D I_{1}\left(\left(\alpha^{2}+j w \mu_{i} \sigma_{i}\right)^{1 / 2} r\right)\right. \\
\left.+E K_{1}\left(\left(\alpha^{2}+j w \mu_{i} \sigma_{i}\right)^{1 / 2} r\right)\right] d \alpha
\end{aligned}
$$

The subscripts of 1,2,3,4 are the permeability and the conductivity of the region II, IV, VI, VIII respectively. The $\alpha$ refers to the separation 'constant' and $B, C, D$ and $E$ are functions of $\alpha$ to be determined. $J_{l}, Y_{l}, I_{l}$ and $K_{l}$ are firstorder Bessel functions [46]. Multi-turns coil in the region I are considered as the superimposition of infinite single turn coil to analyze conveniently. In addition, the region I is also separated the region $I_{0}$ and the region $I_{1}$ in the horizontal part of the shield.

The normal and tangential boundary conditions between the different regions are expressed as follow:

Region I-II/VIII:

$$
\begin{aligned}
& {\left.\left[\begin{array}{c}
A_{\mathrm{I}_{0}}(r, z)=A_{\mathrm{I}_{1}}(r, z) \\
\frac{\partial A_{\mathrm{I}_{0}}}{\partial z}=\frac{\partial A_{\mathrm{I}_{1}}}{\partial z}-\mu_{0} i \delta\left(r-r_{i}\right)
\end{array}\right]\right|_{0<z<z_{0}, r_{1}<r_{i}<r_{2}}} \\
& {\left.\left[\begin{array}{c}
A_{\mathrm{I}}(r, z)=A_{\mathrm{II}}(r, z) \\
\frac{1}{\mu_{0}} \frac{\partial A_{\mathrm{I}}}{\partial z}=\frac{1}{\mu_{1}} \frac{\partial A_{\mathrm{II}}}{\partial z}-i \delta\left(z-z_{i}\right)
\end{array}\right]\right|_{r=r_{2}, 0<z_{i}<z_{0}}} \\
& {\left[\begin{array}{l}
A_{\mathrm{I}_{0} / \mathrm{I}_{1}}(r, z)=A_{\mathrm{II} / \mathrm{VIII}}(r, z) \\
\left.\frac{1}{\mu_{0}} \frac{\partial A_{\mathrm{I}_{0} / \mathrm{I}_{1}}}{\partial z}=\frac{1}{\mu_{1 / 4}} \frac{\partial A_{\mathrm{II} / \mathrm{VIII}}}{\partial z}\right]\left.\right|_{z=z_{0} / 0, r_{1}<r<r_{2}}
\end{array}\right.}
\end{aligned}
$$

The sign ' $/$ distinguishes boundary conditions in different regions. The rest of regions obey a similar method as above. Therefore, the induced voltage of pick-up coil is given as:

$$
V_{\text {pick-up }}=\sum_{w=0}^{\infty} \frac{j 2 \pi w n}{z_{0}\left(r_{2}-r_{1}\right)} \int_{0}^{z_{0}} \int_{r_{1}}^{r_{2}} r A d r d z
$$

where $n$ denotes the number of turns for pick-up coil, $z_{0}\left(r_{2}-\right.$ $r_{l}$ ) denotes the cross section of the coil and $w$ (omega) is angular frequency. It can be regarded as pulse wave as the superimposition of infinite angular frequency. 


\section{EXPERIMENT SET-UP}

\section{A. Numerical simulation}

In order to confirm the performance of the proposed sensor structure, the simulation study is conducted. COMSOL Multiphysics as professional FEM simulation software is applied to model the RFECT probe [42]. It is directly used for FEM calculation and it does not require to input the boundary conditions in which the solving coefficients can be calculated automatically. Calculated results can be analyzed with several function in the software which including plot, Fourier transform, relationships of electromagnetic field among variables and etc. This study mainly focuses on the detectability on cracks of depth and width in ferromagnetic material. All of simulation results are on the basis of $2 \mathrm{D}$ axisymmetric solution, where the inclined defects could not be built due to the limitation of 2D simulation. Although 3D simulation can be applied to model the inclined defects, it will cause a huge computational burden due to the complex mesh generation which decreases the precision of calculation. In addition, complicated 3D model increase the complexity of solutions which reduces the reliability of solution as well.

Fig. 3 (a) shows the diagram of the sensor, which contains the tested ferromagnetic plate and multi-layer magnetic focusing RFEC probe, the detailed probe structure displayed from a sectional view is shown in Fig. 3 (b). Fig. 3 (c) shows the mesh diagram where the mesh in the vicinity of the probe is intensive and it is closely related to the solution region. Fig. 3 (d) shows the probe dimension of $2.4 \mathrm{~cm}$ in radius. The red dotted line in Fig3 (d) bisects the respective shielding layer to two rectangles for describing the size of shield conveniently. Table I reflects the parameters of model, magnetic shielding materials comprise of Iron and Aluminum. The 3D model is shown because that could display the proposed RFEC model clearly and this will be easily interpretation.

1) Impaction analysis of magnetic shielding layer: Firstly, the probe with the shield absent and with the shield applied are built, it consists of the flat conductor, driver coil, the first layer shield, the second layer shield, the third layer shield and air domain. The probe with only the driver coil and the RFEC probe with multi-layer shields are studied to analyze the impact of magnetic shielding layer, the simulation results are shown in Fig4. Poynting vector is mainly used to describe the whole distribution of the direct and indirect coupling energy on the specimen in this model as this can help us to easily evaluate whether the energy can penetrate twice for obtaining RFEC phenomenon. The shielding is a comprehensive process who indeed consist of coupling of magnetic and eddy current in shielding zone. The parameters of simulation are shown in Table I. The driver frequency is $95 \mathrm{~Hz}$, and driver current is $0.3 \mathrm{~A}$. Fig. 4 (a) shows the energy (Poynting vector) distribution, the direct coupling energy upon the plate is dominant, yet the indirect energy does not penetrate the plate at all. Fig. 4 (b), Fig. 4 (c) and Fig. 4 (d) are the energy distribution refer successively to apply 1-layer, 2-layer and 3-layer shields, the indirect coupling energy upon the plate can be observed where it is more priority than the direct with increasing the number of shielding layers. In addition, it is apparently observed for the energy to penetrate the plate and to return back the surface in Fig. 4 (d). It illustrates that the shielding effectiveness of multi-layer shields is better than the individual shield [34]. The whole probe structure for the concentration of direct coupling energy where it is produced by the driver coil in which is constrained at the proximity of the shielding layer. For further affirming the phenomenon of the remote field eddy current, the logarithmic amplitude of magnetic field perpendicular component and the phase are studied. A potential valley and $180^{\circ}$ phase change can be observed as shown in Fig. 5 (a), the amplitude knee point and the phase knot which are the typical characteristics of RFEC [41]. Hence, it is confirmed that the RFEC phenomenon is arisen from the planar sheet.

The effectiveness of Magnetic shielding is an important factor for achieving remote field eddy current, the 2D cutline which denotes transversal is chosen to analyze the change of the z-direction magnetic field, and a featured point is used to evaluate the magnetic shielding effectiveness as shown in Fig. 3 (d).

Fig. 5 (b) displays the energy of upon the plate which is weaker than that under the plate. The indirect energy is richer than that in the direct vicinity of the transversal. In addition, the direct energy is suppressed well. The shielding effectiveness of the feature point is calculated by using the Eq. (1) which is shown in Table II. The shielding effectiveness is gradually strengthened by increasing the shielding layers.

Table I. Parameters of simulation

\begin{tabular}{cccccc}
\hline & $\begin{array}{c}\text { Driver/Pick- } \\
\text { up coil }\end{array}$ & Specimen & $\begin{array}{c}\text { The 1st layer } \\
\text { shield(1/2) }\end{array}$ & $\begin{array}{c}\text { The 2nd layer } \\
\text { shield(3/4) }\end{array}$ & $\begin{array}{c}\text { The 3rd layer } \\
\text { shield(5/6) }\end{array}$ \\
\hline Width(m) & 0.002 & 0.8 & $0.012 / 0.004$ & $0.018 / 0.004$ & $0.024 / 0.004$ \\
Height(m) & 0.01 & 0.01 & $0.002 / 0.01$ & $0.002 / 0.013$ & $0.002 / 0.018$ \\
Conductivity(S/m) & & $5.5 \mathrm{e} 6$ & $1.12 \mathrm{e} 7$ & $3.774 \mathrm{e} 7$ & $1.12 \mathrm{e} 7$ \\
Relative permeability & 190 & 4000 & 1 & 4000 \\
$\quad \begin{array}{l}\text { Turns } \\
\text { AWG }\end{array}$ & $400 / 1000$ & & & & \\
\hline
\end{tabular}
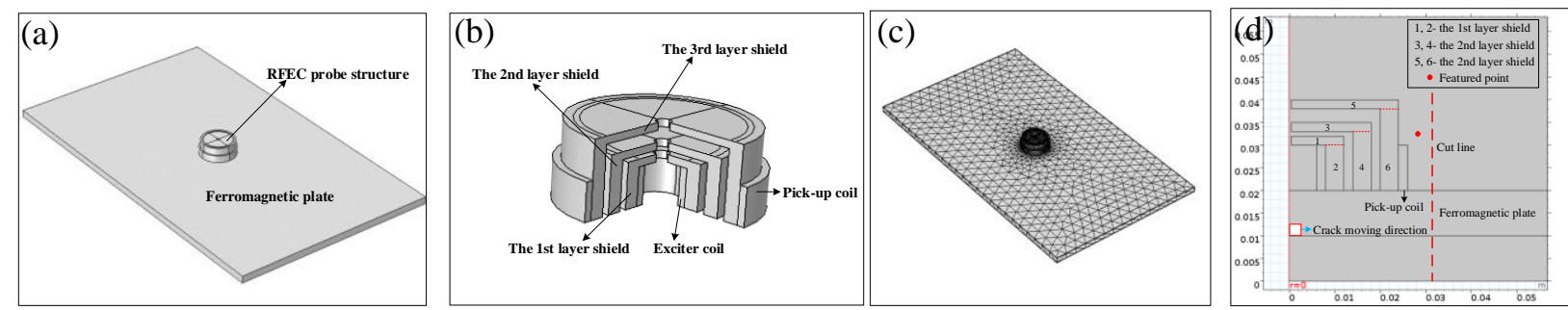

Fig. 3 (a) Space diagram (b) sectional view of the probe (c) FEM mesh diagram (d) 2D axisymmetric view 

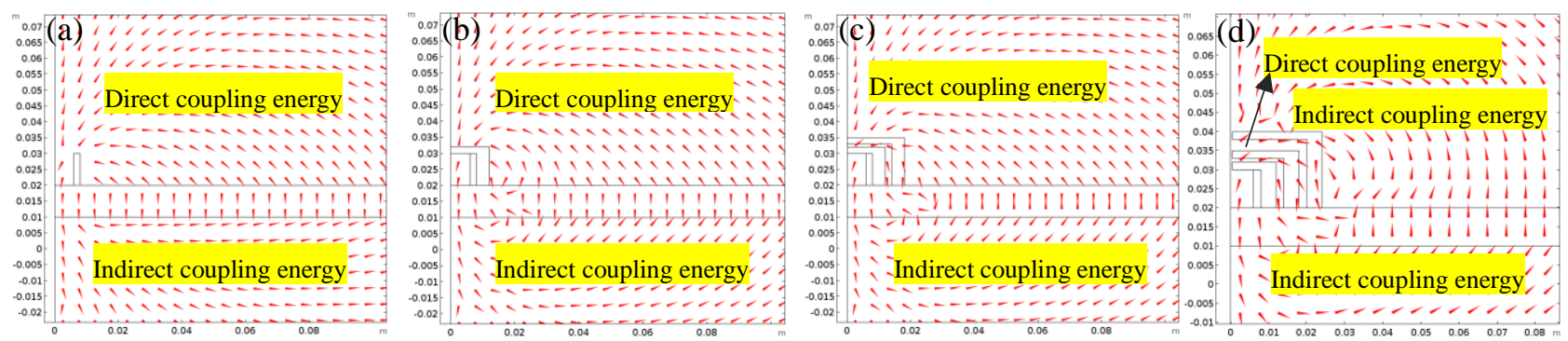

Fig. 4 (a) Poynting vector distribution without shielding units (b) Poynting vector with 1-layer shield (c) Poynting vector with 2-layer shields (d) Poynting vector with 3-layer shields
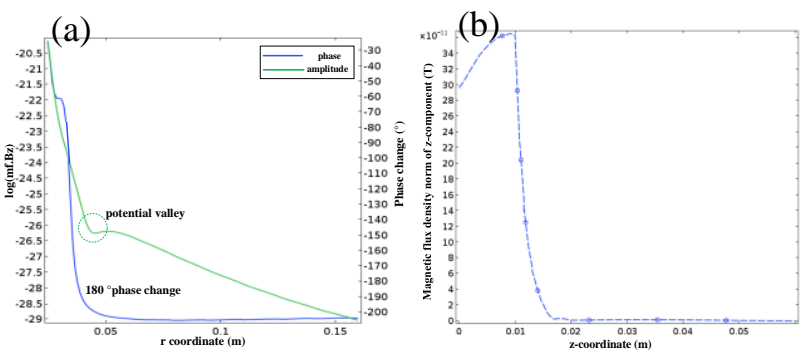

Fig. 5 (a) Logarithmic amplitude and change of phase (b) Magnetic flux density norm of z-component in the cut-line

Table II. SE (dB) in the feature point

\begin{tabular}{ccc}
\hline & Magnetic flux density norm $(\mathrm{T})$ & $\mathrm{SE}(\mathrm{dB})$ \\
\hline no shield & $1.3291 \mathrm{e}-5$ & 0 \\
1-layer shield & $1.2031 \mathrm{e}-8$ & 140 \\
2-layer shield & $7.9203 \mathrm{e}-11$ & 241 \\
3-layer shield & $8.1193 \mathrm{e}-12$ & 286 \\
\hline
\end{tabular}

2) Effect of mode excitation: In the proposed method, it is noted that the frequency is essential for RFEC phenomenon and required to search properly value. Therefore, a rich frequency spectrum of pulse excitation is investigated. Its wide frequency can adjust the selection of proper RFEC frequency. The follow function is conducted to produce pulse of $10 \mathrm{~ms}$ duration beginning at $10 \mathrm{~ms}$ and with a $10 \mu \mathrm{s}$ rise time. The function flc2hs is a smooth Heaviside function with a continuous second derivative without overshoot from COMSOL Multiphysics, namely

$$
1.2[f l c 2 h s(t-0.01,1 e-5)-f l c 2 h s(t-0.02,1 e-5)]
$$

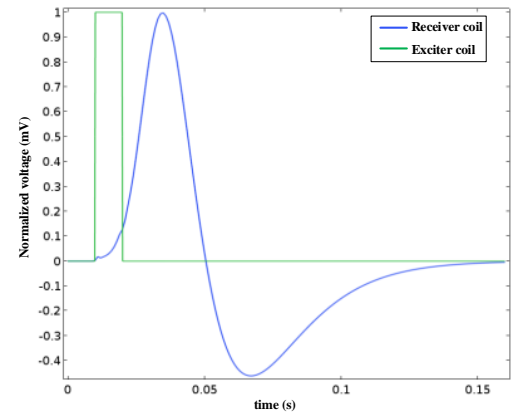

Fig. 6 Normalized voltage of driver coil and receiver coil

Fig. 6 is the normalized voltage curve of the driver and the receiver coil $[19,25]$. Comparing with the traditional pulse response, there is a duration time between the rising edge and the falling edge in pulse excitation which causes the output response is identical interval. However, that duration does not exist in pulsed remote field eddy current. It can be observed that the response of PRFEC is apparently lag the excitation pulse time due to the diffusion of the indirect coupling energy results $[20,26]$.
3) Impaction analysis of the relationship between position and sensitivity: In Fig. 3 (d), the crack of $2 \mathrm{~mm} \mathrm{x}$ $2 \mathrm{~mm}$ in dimension moves along $r$-coordinate positive axis from the current position to $r=0.05 \mathrm{~m}$ with a $0.026 \mathrm{~m}$ interval. The crack is identical located at different $r$ coordinate for obtaining optimal sensitivity of the measurement, the sensitivity of testing refers to the follow equation,

$$
S=\frac{\left|\left(V_{(\text {crack }) \max }-V_{(\text {no-crack }) \max }\right)\right|}{V_{(\text {no-crack }) \max }}
$$

where $S$ is sensitivity of crack in corresponding place, the subscripts of (crack) $\max$ and (no-crack) $)_{\max }$ refer successively to the maximum absolute value of sensing coil voltage when the crack existent and inexistent.

The voltage amplitudes of the pick-up coil will be increased when the crack locates in the proximity of the second and third shielding layer, and decreased when the crack gradually away from the probe, as shown in Fig. 7 (a). It can be seen that the sensitivity reaches the maximum in which the crack at the $r=16 \mathrm{~mm}$ from the Fig. 7 (b). Hence, the region will be represented as the most sensitive position for studying variations of the crack height and the crack width.
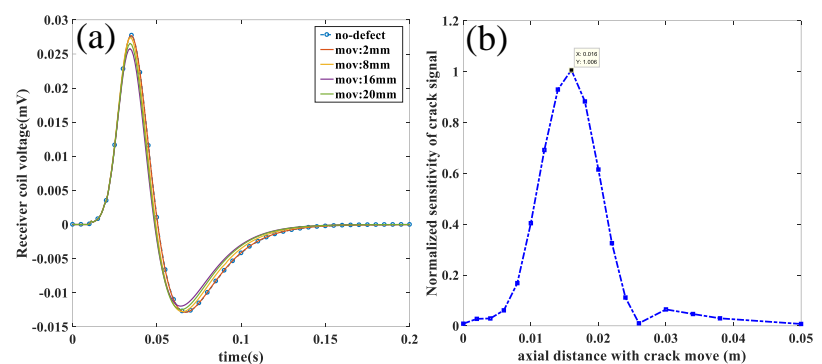

Fig. 7 (a) Pick-up voltage with crack move (b) Normalized sensitivity of crack

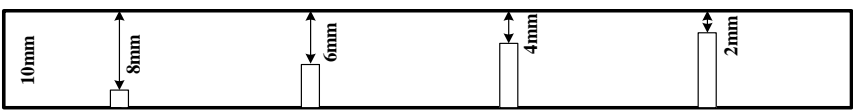

Fig. 8 Variations of the sub-surface crack height

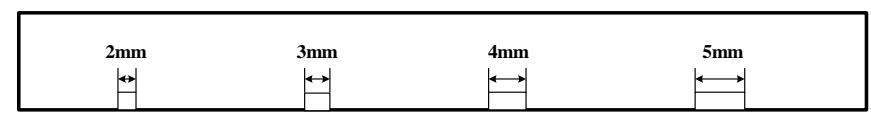

Fig. 9 Variations of the sub-surface crack width

4) Impaction of sub-surface and surface defects: The plates of $10 \mathrm{~mm}$ in thickness below are used and variations of the crack are shown in Fig. 8 and Fig. 9, respectively.

Fig. 10 (a) is a differential voltages which are subtracted the crack absent voltage from the crack voltage. We can extract the maximum absolute values of the first peak to quantitative study a relationship between the crack height 
and the response. A linear relation can be observed in Fig.
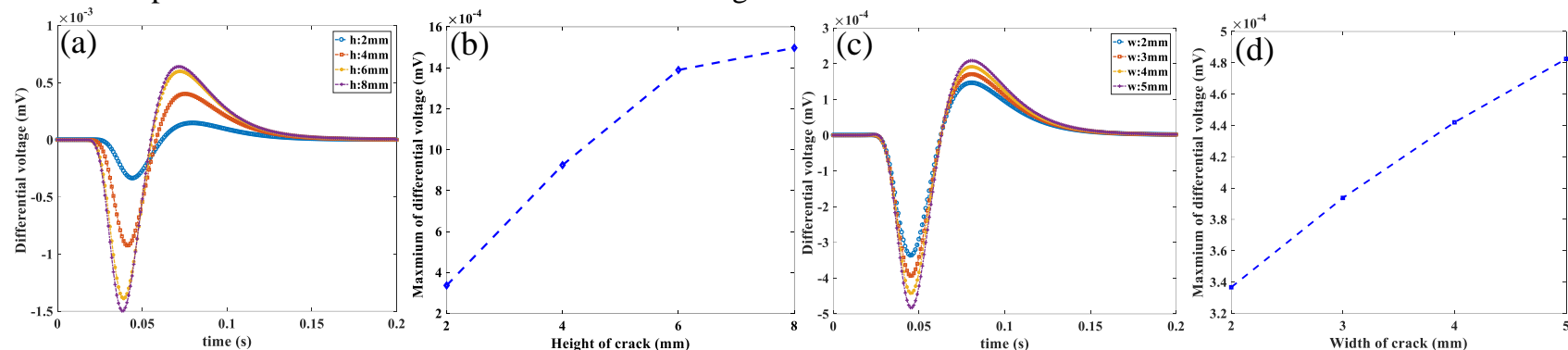

Fig. 10 Sub-surface: (a) Differential voltage among different crack height (b) Maximum of differential voltage with changing the height of the crack (c) Differential voltage among different crack width (d) Maximum of differential voltage with changing the width of the crack
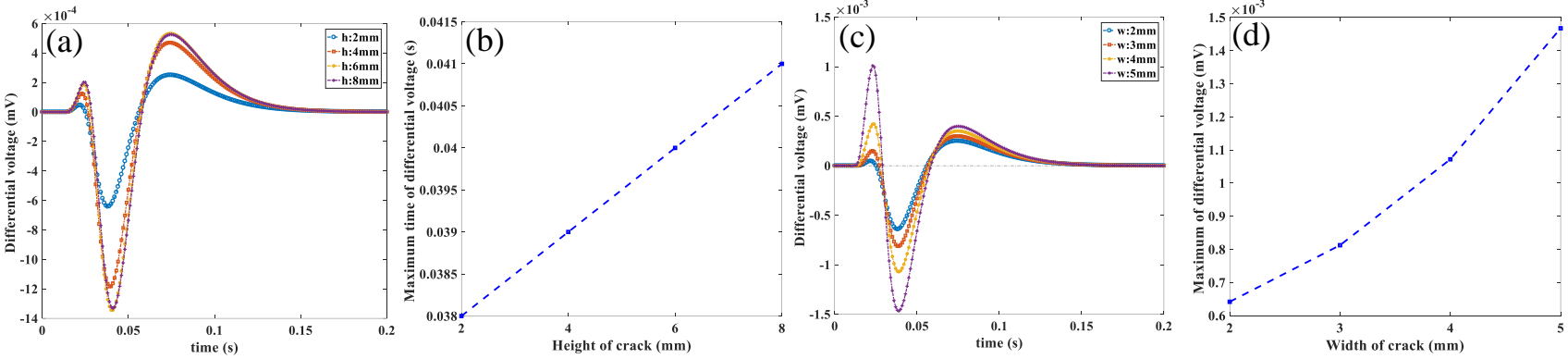

Fig. 11 Surface: (a) Differential voltage among different crack height (b) Maximum time of differential voltage with changing the height of the crack (c) Differential voltage among different crack width (d) Maximum of differential voltage with changing the width of the crack

10 (b). Thus, it can be confirmed that the height of the crack acquire from the response signal. Fig. 10 (c) shows the differential response with different width, a better linear relation is shown in Fig. 10 (d). It indicates the width of the crack can be more accurately detected and can be applied to quantitative evaluate the dimension of the defect. The specimens above are inverted as surface defects for inspection. Fig. 11 (a) shows the differential voltages of the surface crack with different height, the second peak time is applied to rate surface cracks in Fig. 11 (b).Fig. 11 (c) and Fig. 11 (d) exhibit individually the relationship between the height of crack and the response of pick-up coil, the maximum absolute value of the second peak is used to assess different surface cracks width. The peak, the peak time and the zero-crossing time and so on in the figure above can be applied as characteristic values to assess the sizes of the crack [45].

\section{B. Experimental validation}

1) Experimental platform and specimen defects: For the experiment verification, the proposed experimental platform of PRFEC is generated as shown in Fig. 12. The probe consists of driver coil of 400 turns, pick-up coil of 1000 turns, and three layer shields with Iron and Aluminum individually. A specimen of 45\# steel with three groups of defects is used. The defects with different width, height and shape are inspected, a signal processing circuit combined with AD620 and AD623 instrument amplifier, the filter circuit including a single DC power converted to positive and negative DC power to supply the low-pass filter with a bandwidth of $100 \mathrm{~Hz}$. The amplitude of exciting pulse is $10 \mathrm{~V}$, the cycle is $40 \mathrm{~ms}$ with a $4 \mathrm{~ms}$ pulse width. A difference between the simulation and experiment has been discovered in the actual experiment due to size of the probe as material parameters are not exactly same. Hence A pulse of $4 \mathrm{~ms}$ duration has a preferable response while with a $10 \mathrm{~ms}$ duration. When a cycle pulse produced from the signal generator and amplified by a power amplifier through the driver coil, the pick-up coil connected with two signal amplifier of AD620 and AD623, and the amplified signal goes across the low-pass filter eventually to filter several perturbation, the processed receiving signal can be directly observed and obtained from an oscilloscope.

Fig. 13 shows all kinds of surface defects in a sample with a thickness of $10 \mathrm{~mm}$, the reverse side of the specimen is viewed as the sub-surface defects, The rest of cracks are a length of $20 \mathrm{~mm}$ and a width of $2 \mathrm{~mm}$ except for circle defects and defects in $3 \mathrm{~mm}$ and $4 \mathrm{~mm}$ width as shown in Fig13, where three cracks in a depth of $5 \mathrm{~mm}$ with distinguish slope angle of $30^{\circ}, 45^{\circ}, 60^{\circ}$. Three different circle defects in a depth of $4 \mathrm{~mm}$ with diameter in $5 \mathrm{~mm}$, $7 \mathrm{~mm}$ and $10 \mathrm{~mm}$, three rectangular cracks with depth of $4 \mathrm{~mm}, 6 \mathrm{~mm}$ and $8 \mathrm{~mm}$ and three rectangular cracks in a depth of $2 \mathrm{~mm}$ with different width of $2 \mathrm{~mm}, 3 \mathrm{~mm}$ and $4 \mathrm{~mm}$. The defects above will be analyzed in the next section.

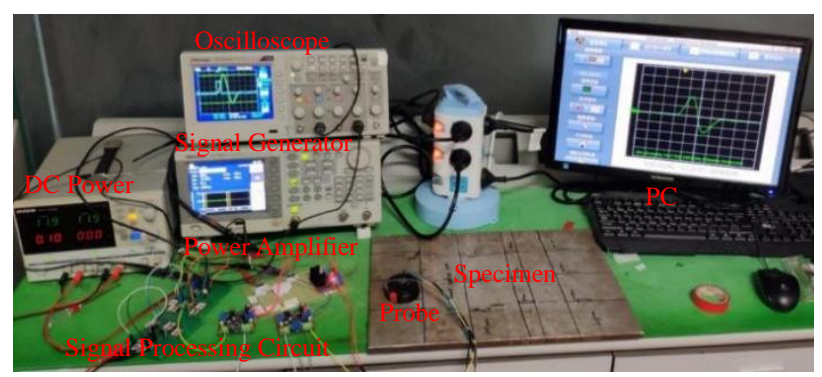

Fig. 12 Experiment platform of PRFEC

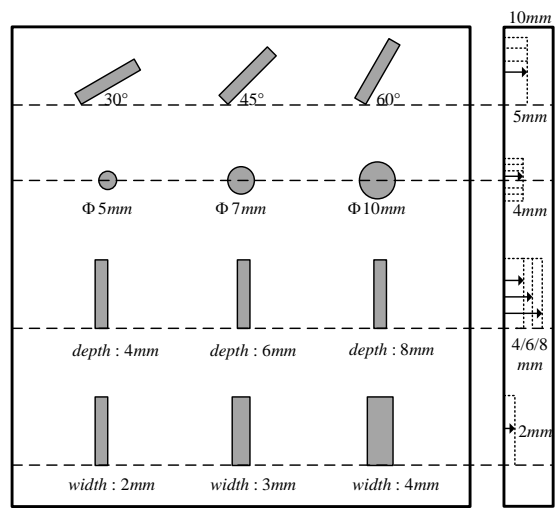


Fig. 13 Front view and side view of diverse defects in specimen
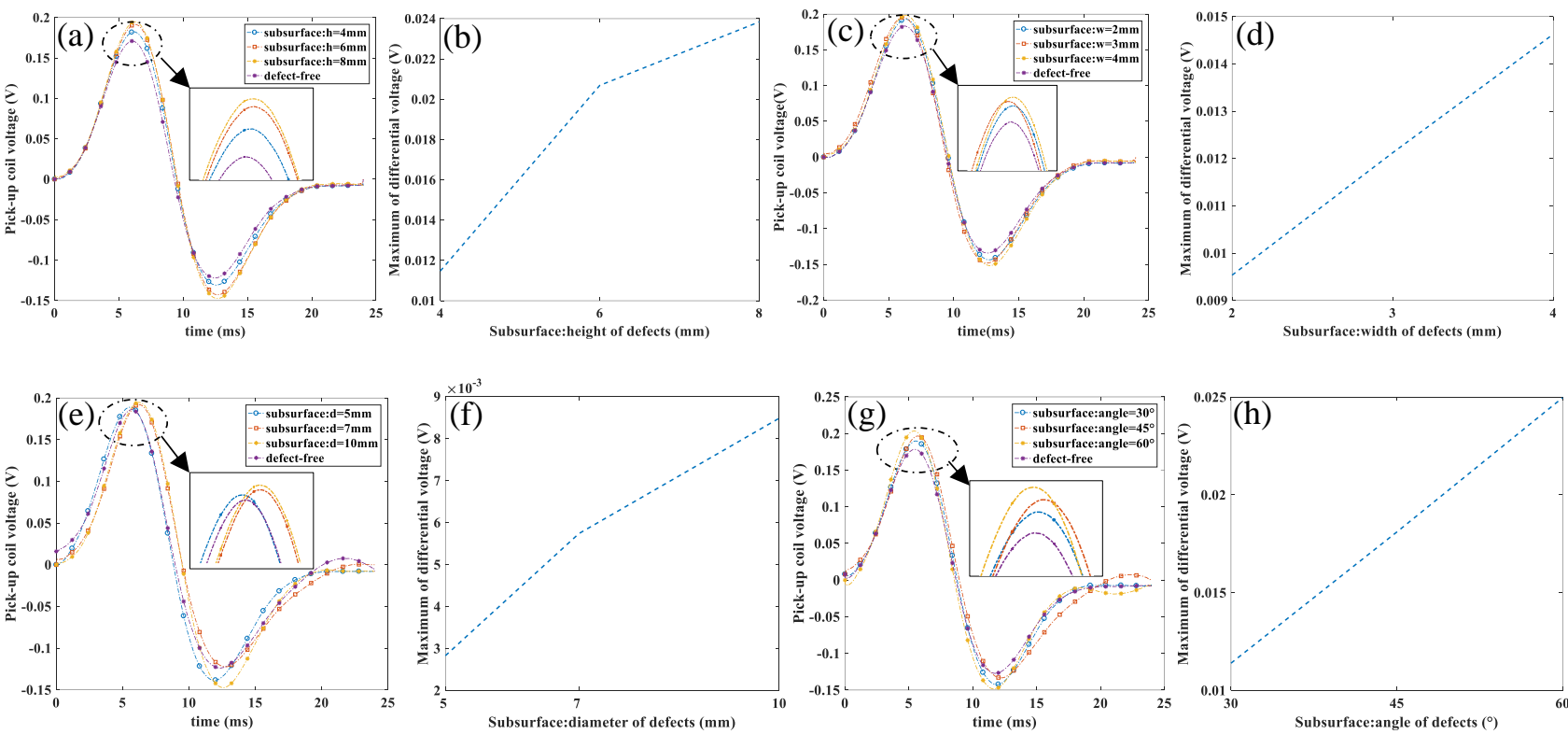

Fig. 14 Sub-surface: (a) Pick-up voltage in different defect height (b) Maximum of differential voltage with changing the height of the crack (c) Pick-up voltage in different defect width (d) Maximum of differential voltage with the various width of the crack (e) Pick-up voltage in different diameter of circular defect (f) Maximum of differential voltage with the various diameter of the crack (g) Pick-up voltage in different angle of defect (h) Maximum of differential voltage with varied angle
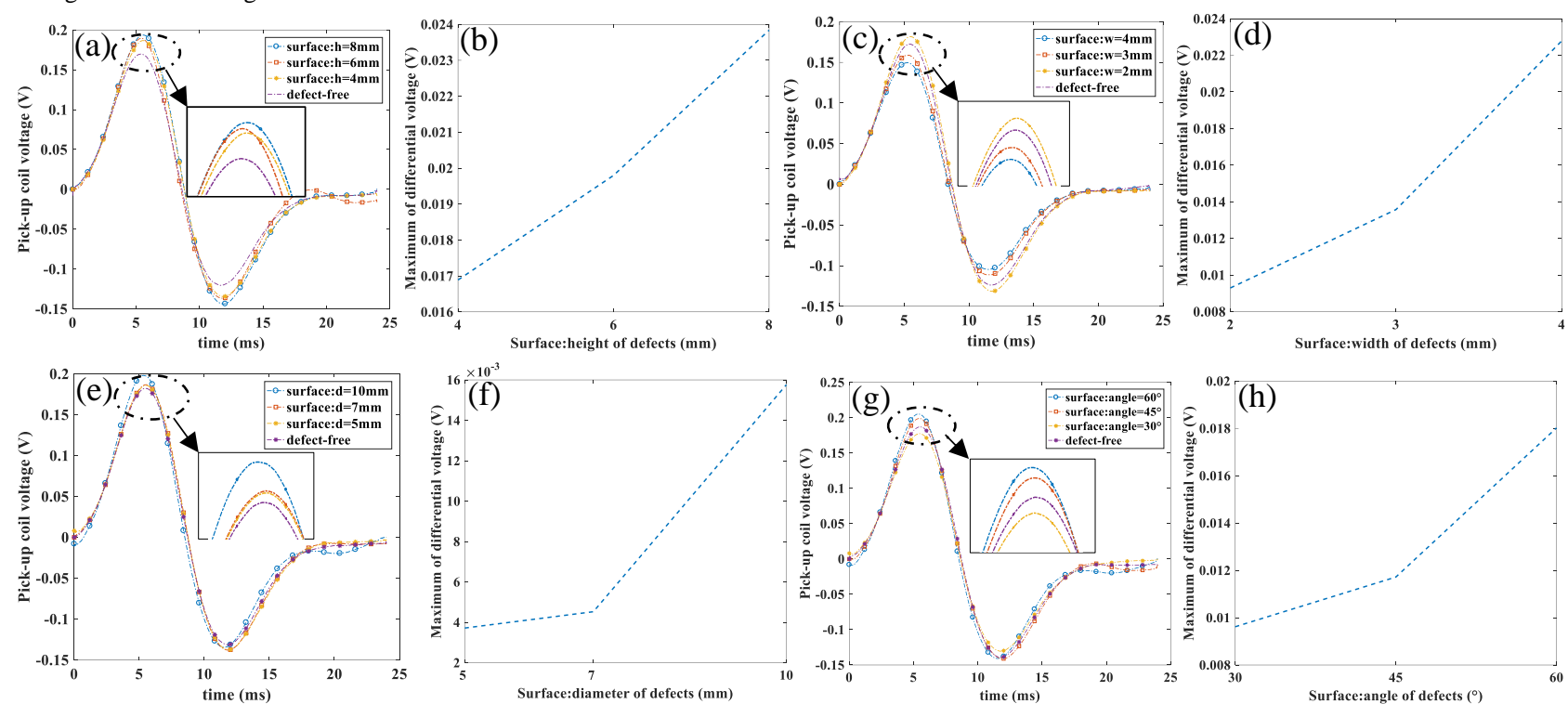

Fig. 15 Surface: (a) Pick-up voltage in different defect height (b) Maximum of differential voltage with changing the height of the crack (c) Pick-up voltage in different defect width (d) Maximum of differential voltage with the various width of the crack (e) Pick-up voltage in different diameter of circular defect (f) Maximum of differential voltage with the various diameter of the crack (g) Pick-up voltage in different angle of defect (h) Maximum of differential voltage with varied angle

Table III. Correlation coefficients between parameters of defect and sensitivity

\begin{tabular}{|c|c|c|c|c|c|c|}
\hline \multirow{2}{*}{\multicolumn{3}{|c|}{ Parameters of defect }} & \multicolumn{2}{|c|}{ Sensitivity } & \multicolumn{2}{|c|}{ Correlation coefficients } \\
\hline & & & ace & & Subsurface & Surface \\
\hline \multicolumn{3}{|c|}{ Height of defect $(\mathrm{mm})$} & \multirow{2}{*}{$0.0671,0.121,0.1395$} & \multirow{2}{*}{$0.0995,0.1166,0.1404$} & \multirow[b]{2}{*}{0.9622} & \multirow[b]{2}{*}{0.9956} \\
\hline 4 & 6 & 8 & & & & \\
\hline \multicolumn{3}{|c|}{ Width of defect $(\mathrm{mm})$} & \multirow{2}{*}{$0.0520,0.0661,0.0798$} & \multirow{2}{*}{$0.0539,0.0788,0.1322$} & & \multirow[b]{2}{*}{0.9785} \\
\hline 2 & 3 & 4 & & & & \\
\hline \multicolumn{3}{|c|}{ Diameter of Circular defect $(\mathrm{mm}$} & \multirow[b]{2}{*}{$0.0152,0.0308,0.0456$} & \multirow[b]{2}{*}{$0.0204,0.0248,0.0865$} & \multirow[b]{2}{*}{0.9914} & \multirow{2}{*}{0.9399} \\
\hline 5 & 7 & 10 & & & & \\
\hline \multicolumn{3}{|c|}{ Angle of def } & & & & \\
\hline 30 & 45 & 60 & & & 1 & \\
\hline
\end{tabular}

2) Experimental result analysis: All defects are detected where the scanning direction is perpendicular to the defects which are vertical. A best group of data are obtained to reflect the responses and the relationships between the types of defects and the features. The maximum value of the first peak in differential voltage is regarded as a feature to exhibit the relation of different types of defects.

Fig. 14 shows the receiver signals of different height, width, diameter and angle of defects in sub-surface defects detection. In Fig. 14 (a) and (b), a linear relation can be observed with alternation of the height of the defect. The magnitude of the pick-up voltage with defect is bigger than that in the defect free region. From Fig. 14 (c) and (d), the sensing voltage will increase with the increment of defect width, a better linear relationship is generated. Fig. 14 (e) and (f) reflect a response from circular defect, the pick-up voltage will strengthen when diameter of defects from $5 \mathrm{~mm}$ to $10 \mathrm{~mm}$ beneath $6 \mathrm{~mm}$ from the surface. From $30^{\circ}$ to $60^{\circ}$, the contact area will be enlarged, the pick-up voltage will be magnified in Fig. 14 (g) and (h), respectively.

As shown in Fig. 15 (a)-(h), a diverse of surface defects can be inspected, the results indicate a preferable linear 
relationship between the pick-up voltage and defects. In Fig. 15 (a)-(b), the height of defect is analyzed, the receiver response will be enlarged when the height from $4 \mathrm{~mm}$ to $8 \mathrm{~mm}$ because of the defects have a bigger air-gap that can block the eddy current. From Fig. 15 (g) and (h), the defects owing to different inclined angle occur the different eddy current flows will alter the receiving response. Table III shows the correlation coefficients between the parameters of defect and sensitivity. Table III summarizes the sensitivity of the proposed system for all kind of defects. It indicates that the sensitivity of the detection has highly correlation with the characteristic of the defects. It approximately reaches to one on average.

From the above results and numerical simulation, the experiment validation agrees well with the simulation. One interpretation of experiment which pick-up response has apparent phenomenon that is not duration between the rising and falling edge of pulse. It approximates a sin wave as a result of magnetic shielding effectiveness and energy propagation. The proposed sensor structure can be applied to inspect both sub-surface and surface cracks of ferromagnetic material. It can inspect the defect below the surface of $8 \mathrm{~mm}$ and this is sensitive for rectangular, circular and inclined cracks.

\section{CONCLUSION AND FUTURE WORK}

In this paper, we have proposed a multi-layer magnetic focusing sensor framework with a driver coil, pick-up coil and three layer shields with different shielding materials. It is based on magnetic shielding theory to achieve RFEC phenomenon in plate. The proposed work obvious that the energy produced by excitation coil propagates from the surface of plate to the below of the surface and back to the surface. A set of surface and subsurface cracks are tested and validated by using the proposed method and it is evidenced that all works have a good reaction among the response and multivariable defects. The smallest size of the crack with a width of $2 \mathrm{~mm}$ and length of $20 \mathrm{~mm}$ which is beneath $8 \mathrm{~mm}$ from the surface is able to be detected. A number of circular defects and inclined cracks are confirmed to match a better detectability. Future works will focus on the test sensitivity, lift-off effect and deeper subsurface defects.

\section{ACKNOWLEDGEMENT}

The work was supported by state administration of quality supervision, inspection and quarantine (No. 2017QK042), supported by National Natural Science Foundation of China (No. 61401071, No. 61527803)

\section{REFERENCES}

[1] Sophian A, Tian G Y, Taylor D, et al. Electromagnetic and eddy current NDT: a review[J]. Insight, 2001, 43(5): 302-306.

[2] Topp D, Smith M. Application of the ACFM inspection method to rail and rail vehicles[J]. Insight-Non-Destructive Testing and Condition Monitoring, 2005, 47(6): 354-357.

[3] Papaelias M P, Lugg M C, Roberts C, et al. High-speed inspection of rails using ACFM techniques[J]. NDT \& e International, 2009, 42(4): 328-335.

[4] Li W, Yuan X, Chen G, et al. A feed-through ACFM probe with sensor array for pipe string cracks inspection[J]. NDT \& E International, 2014, 67: 17-23.

[5] Li W, Yuan X, Chen G, et al. High sensitivity rotating alternating current field measurement for arbitrary-angle underwater cracks[J]. NDT \& E International, 2016, 79: 123-131.
[6] Raine A, Lugg M. A review of the alternating current field measurement inspection technique[J]. Sensor Review, 1999, 19(3): 207-213.

[7] Papaelias M, Cheng L, Kogia M, et al. Inspection and structural health monitoring techniques for concentrated solar power plants[J]. Renewable Energy, 2016, 85: 1178-1191.

[8] Park G S, Park E S. Improvement of the sensor system in magnetic flux leakage-type nondestructive testing (NDT)[J]. IEEE Transactions on magnetics, 2002, 38(2): 1277-1280.

[9] Li Y, Tian G Y, Ward S. Numerical simulation on magnetic flux leakage evaluation at high speed[J]. NDT \& E International, 2006, 39(5): 367-373.

[10] Sophian A, Tian G Y, Zairi S. Pulsed magnetic flux leakage techniques for crack detection and characterisation[J]. Sensors and Actuators A: Physical, 2006, 125(2): 186-191.

[11] Singh W S, Rao B P C, Vaidyanathan S, et al. Detection of leakage magnetic flux from near-side and far-side defects in carbon steel plates using a giant magneto-resistive sensor[J]. Measurement Science and Technology, 2007, 19(1): 015702.

[12] Tsukada K, Yoshioka M, Kawasaki Y, et al. Detection of back-side pit on a ferrous plate by magnetic flux leakage method with analyzing magnetic field vector[J]. NDT \& E International, 2010, 43(4): 323-328.

[13] Kasai N, Takada A, Fukuoka K, et al. Quantitative investigation of a standard test shim for magnetic particle testing[J]. NDT \& E International, 2011, 44(5): 421-426.

[14] Atherton D L. Remote field eddy current inspection[J]. IEEE Transactions on Magnetics, 1995, 31(6): 4142-4147.

[15] Fukutomi H, Takagi T, Nishikawa M. Remote field eddy current technique applied to non-magnetic steam generator tubes[J]. NDT \& E International, 2001, 34(1): 17-23.

[16] Lord W, Sun Y S, Udpa S S, et al. A finite element study of the remote field eddy current phenomenon[J]. IEEE Transactions on Magnetics, 1988, 24(1): 435-438.

[17] Hoshikawa H, Saitou H, Koido J, et al. Energy flow in remote field eddy current nondestructive testing[J]. IEEE Transactions on Magnetics, 1990, 26(2): 885-888.

[18] Kim D, Udpa L, Udpa S. Remote field eddy current testing for detection of stress corrosion cracks in gas transmission pipelines[J]. Materials Letters, 2004, 58(15): 2102-2104.

[19] Chen M, Sun Y, Lord W, et al. Pulsed RFEC probe response[J]. IEEE Transactions on magnetics, 1992, 28(2): 1430-1433.

[20] Yang B, Li X. Pulsed remote eddy current field array technique for nondestructive inspection of ferromagnetic tube[J]. Nondestructive testing and evaluation, 2010, 25(1): 3-12.

[21] YANG B, ZHANG H, JING Y, et al. Inspection Principle and Defect Quantitative Estimation of Pulsed Remote Field Eddy Current Technique $[\mathrm{J}][\mathrm{J}]$. Journal of Air Force Engineering University (Natural Science Edition), 2012, 6: 011.

[22] Xu X, Liu M, Zhang Z, et al. A novel high sensitivity sensor for remote field eddy current non-destructive testing based on orthogonal magnetic field[J]. Sensors, 2014, 14(12): 24098-24115.

[23] Luo Q, Shi Y, Wang Z, et al. A Study of Applying Pulsed Remote Field Eddy Current in Ferromagnetic Pipes Testing[J]. Sensors, 2017, 17(5): 1038.

[24] Vasic D, Bilas V, Ambrus D. Pulsed eddy-current nondestructive testing of ferromagnetic tubes[J]. IEEE transactions on instrumentation and measurement, 2004, 53(4): 1289-1294.

[25] Vasić D, Bilas V, Ambruš D. Measurement of ferromagnetic tube wall thickness using pulsed remote field technique[C]//Proceedings of the 12th IMEKO TC4 International Symposium Electrical Measurements and Instrumentation, Zagreb, Croatia. 2002: 25-27.

[26] Vasić D, Bilas V, Ambruš D. Pulsed Remote Filde Technique in Ferromagnetic Tube Wall Thickness and Inner Diameter Measurement[C]//XVII IMEKO World Congress. 2003: 912-916.

[27] Dadić M, Vasić D, Bilas V. A system identification approach to the modelling of pulsed eddy-current systems[J]. NDT \& E International, 2005, 38(2): 107-111.

[28] Thirunavukkarasu S, Rao B P C, Jayakumar T, et al. Techniques for processing remote field eddy current signals from bend regions of steam generator tubes of prototype fast breeder reactor $[\mathrm{J}]$. Annals of Nuclear Energy, 2011, 38(4): 817-824.

[29] Kasai N, Fujiwara Y, Sekine K, et al. Evaluation of back-side flaws of the bottom plates of an oil-storage tank by the RFECT[J]. NDT \& E International, 2008, 41(7): 525-529.

[30] Kasai N, Matsuzaki S, Sakamoto T. Experimental and analytical study for detectability of the back-side flaws of flat ferromagnetic plates by RFECT[J]. Ndt \& E International, 2011, 44(8): 703-707.

[31] Wang J, Yusa N, Pan H, et al. Evaluation of sensitivity of remote field eddy current testing and low-frequency eddy current testing 
for inspecting grooves of metal plate[J]. Materials Transactions, 2013, 54(1): 90-95

[32] Yang B, Xu J, Wu H, et al. Magnetic field shielding technique for pulsed remote field eddy current inspection of planar conductors[J]. NDT \& E International, 2017, 90: 48-54.

[33] Kobayashi N, Ueno S, Nagai S, et al. Remote field eddy curren testing for steam generator inspection of fast reactor[J]. Nuclear Engineering and Design, 2011, 241(12): 4643-4648.

[34] Hasselgren L, Luomi J. Geometrical aspects of magnetic shielding at extremely low frequencies[J]. IEEE transactions on Electromagnetic Compatibility, 1995, 37(3): 409-420.

[35] Sandrolini L, Massarini A, Reggiani U. Transform method for calculating low-frequency shielding effectiveness of planar linear multilayered shields[J]. IEEE transactions on magnetics, 2000, 36(6): 3910-3919.

[36] Trenkler Y, McBride L E. Shielding improvement by multi-layer design[C]//Electromagnetic Compatibility, 1990. Symposium Record., 1990 IEEE International Symposium on. IEEE, 1990: 1-4

[37] Schulz R B, Plantz V C, Brush D R. Shielding theory and practice[J]. IEEE Transactions on Electromagnetic Compatibility, 1988, 30(3): 187-201.

[38] Shin Y K. Design of encircling remote field eddy-current probe[J]. IEEE transactions on magnetics, 2002, 38(2): 1273-1276.

[39] Zhou D, Wang J, He Y, et al. Influence of metallic shields on pulsed eddy current sensor for ferromagnetic materials defect detection[J]. Sensors and Actuators A: Physical, 2016, 248: 162-172.

[40] Liu Z, Yao J, He C, et al. Development of a bidirectional-excitation eddy-current sensor with magnetic shielding: Detection of subsurface defects in stainless steel[J]. IEEE Sensors Journal, 2018

[41] Sun Y S, Udpa S, Lord W, et al. Inspection of Metallic Plates Using a Novel Remote Field Eddy Current NDT Probe[M]//Review of Progress in Quantitative Nondestructive Evaluation. Springer Boston, MA, 1996: 1137-1144

[42] Sun Y S, Udpa L, Udpa S, et al. A novel remote field eddy curren technique for inspection of thick-walled aluminum plates[J]. Materials evaluation, 1998, 56(1): 94-97.

[43] Wang H, Luo Q, Wang X, et al. Simulation and experimental study of remote field eddy current testing on flat conductive plate[J]. International Journal of Applied Electromagnetics and Mechanics, 2010, 33(3, 4): 1261-1266.

[44] Multiphysics C. v. 5.2[J]. COMSOL AB, Stockholm, Sweden, 2015.

[45] He Y, Luo F, Pan M, et al. Defect classification based on rectangular pulsed eddy current sensor in different directions[J]. Sensors and Actuators A: Physical, 2010, 157(1): 26-31

[46] Dodd C V, Deeds W E. Analytical solutions to eddy - current probe - coil problems $[J]$. Journal of applied physics, 1968, 39(6): 2829-2838

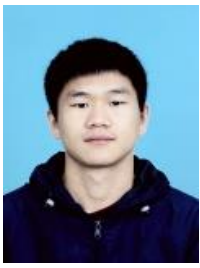

Changrong Yang received the B.Sc. degree in Measurement and control technology and instrument from Southwest Petroleum University (2013-2017), Chengdu, China. He is currently pursuing the M.Sc. degree in nondestructive testing using eddy current technique at the University of Electronic Science and Technology of China, Chengdu, China. His research interests include remote field eddy current.

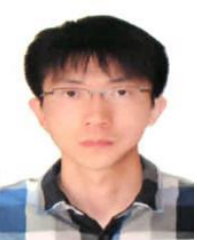

Bin Gao (M' 12-SM' 14) received his B.Sc. degree in communications and signal processing from Southwest Jiao Tong University (2001-2005), China, MSc degree in communications and signal processing with Distinction and $\mathrm{PhD}$ degree from Newcastle University, UK (2006-2011). He worked as a Research Associate (2011-2013) with the same university on wearable acoustic sensor technology. Currently, he is a Professor with the School of Automation Engineering, University of Electronic Science and Technology of China (UESTC), Chengdu, China. His research interests include sensor signal processing, machine learning, social signal processing, nondestructive testing and evaluation where he actively publishes in these areas. He is also a very active reviewer for many international journals and long standing conferences. He has coordinated several research projects from National Natural Science Foundation of China.

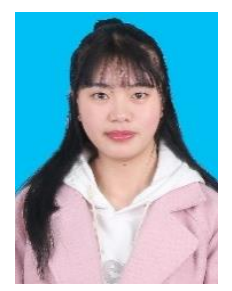

Qiuping Ma received the B.Sc. degree in Electronic and information engineering from Sichuan Normal University (2013-2017), Chengdu, China. He is currently pursuing the M.Sc. degree in nondestructive testing using eddy current technique at the University of Electronic Science and Technology of China, Chengdu, China. His research interests include flexible array eddy current inspection.

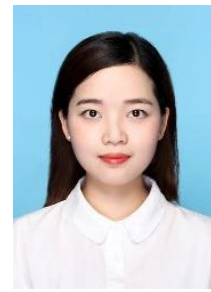

Lian Xie received the B.Sc. degree in Measurement and control technology and instrument from Southwest Petroleum University (2012-2016), Chengdu, China. He is currently pursuing the M.Sc. degree in nondestructive testing using eddy current technique at the University of Electronic Science and Technology of China, Chengdu, China. His research interests include eddy current testing, wireless energy transfer.

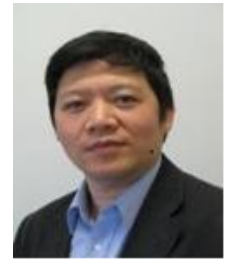

Gui Yun Tian (M'01-SM'03) received the B.Sc degree in metrology and instrumentation and M.Sc degree in precision engineering from the University of Sichuan, Chengdu, China, in 1985 and 1988 , respectively, and the Ph.D. degree from the University of Derby, Derby, U.K., in 1998. From 2000 to 2006, he was a Lecturer, Senior Lecturer, Reader, Professor, and Head of the group of Systems Engineering, respectively, with the University of Huddersfield, U.K. Since 2007, he has been based at Newcastle University, Newcastle upon Tyne, U.K., where he has been Chair Professor in Sensor Technologies. Currently, He is also an adjunct professor with School of Automation Engineering, University of Electronic Science and Technology of China. He has coordinated several research projects from the Engineering and Physical Sciences Research Council (EPSRC), Royal Academy of Engineering and FP7, on top of this he also has good collaboration with leading industrial companies such as Airbus, Rolls Royce, BP, nPower, Networkrail and TWI among others.

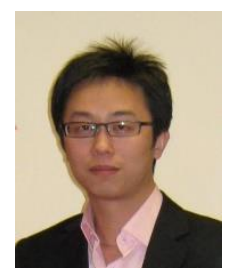

Ying Yin received the BEng degree in Electrical Engineering from Xihua University in 2005, PhD degree in Digital Image Processing from Newcastle University in 2009. He is currently a Senior Engineer and Director of Research Department of Sichuan Special Equipment Inspection Institute. His major research includes non-destructive testing and evaluation for specia equipment specifically, image processing. 